\title{
Review of Peridynamics: Theory, Applications, and Future Perspectives
}

\author{
Gábor Ladányi ${ }^{1}$ - Viktor Gonda ${ }^{2}$ \\ ${ }^{1}$ University of Dunaújváros, Institute of Engineering Sciences, Hungary \\ 2Óbuda University, Donát Bánki Faculty of Mechanical and Safety Engineering, Hungary
}

The modelling and simulation of material degradations, particularly fractures in solids of different lengths and time scales, remains challenging despite the numerous approaches that have been developed. In this review, the focus is set on research work concerned with a very promising non-local method: peridynamic modelling. With this approach, continuous phenomena may be described, and the complete evolution (i.e., initiation, propagation, branching, or coalescence) of cracks and other discontinuities can be followed in solids in an integrated framework. Evaluating the large number of publications on this topic, the authors chose to present concisely the key concepts, applications, and results in identifying possible future paths: the incorporation of mechanics of large deformations and material nonlinearities, and the development of high-efficiency peridynamic solvers. This review does not intersect with recent relevant reviews, which reflects its significance to readers.

Keywords: peridynamics, fracture mechanics, damage mechanics, non-local mechanics

\begin{abstract}
Highlights
- Basic concepts and different branches of peridynamic modelling of crack propagation are introduced.

- The evolution of peridynamic research is assessed based on a wide and representative review of the literature.

- The most frequent fields of applications are highlighted where consensus and contention are reached in modelling.

- Evaluating the features of peridynamic modelling, future research perspectives are highlighted in treating material and geometrical nonlinearities, improving numerical efficiency.
\end{abstract}

\section{INTRODUCTION}

Analytical and numerical methods of continuum physics are the most successful methods in solving engineering problems of solid and fluid mechanics. As the performance of computers has increased, motivated by safety and economic reasons, the field of solvable problems has become wider. New maintenance strategies introduced reliability and riskbased operation methods [1] and [2]. In these methods, the damage of a structure may not lead to the end of its operability. The lifetime ends when reliability decreases under a predefined limit. The definition of this criterion and the estimation of the risk require the analysis of the damaged structure.

Structural failure is a complex phenomenon; it is usually initiated on the micro-scale with microdamage of the material. After the evolution of microdamage, damage appears in the meso- and macro-scales. As this mechanism is in process, the mechanical behaviour of the whole structure may change (e.g., post-buckling behaviour or unstable, brittle failure); sometimes, the change is localized (e.g., ductile failure).

In classical continuum mechanics ( $\mathrm{CCM})$, the continuity and usually the differentiability of the state variables are assumed. As failure involves discontinuities, solving the governing equations defined by CCM with typical computational methods is troublesome [3]. CCM can follow the displacements and strain field as a function of external loads with various material models from simple elasticity to the viscoplastic flow of solids. Limitations of the analytical description are reached when discontinuities appear in any state variables. Continuum damage mechanics can account for the effect of the microcracks and voids through homogenized material properties [4]. Unfortunately, it fails when the size of the damaged zone reaches the macroscale [5].

For an accurate simulation of the fracture process, the numerical method needs to be capable of modelling crack initiation, propagation, bifurcation, coalescence, and separation. Classical linear elastic fracture mechanics (LEFM) or non-linear fracture mechanics (NLFM) may be applied for the loadbearing structures of simple geometry and predefined cracks only. By employing special numerical methods, e.g., extended finite element method (X-FEM) [6] or meshless methods [7], more complicated problems can be solved with acceptable accuracy. The chosen method is acceptable if it is not mesh-dependent. In most cases, the mesh generation at every time step of the solution is necessary to fulfil this requirement [6]. The increased time of mesh generation is a very serious drawback. Additionally, crack initiation and crack branching remain open questions in this context, 
and the crack-tip kinematics has to be controlled with artificial criteria [3].

The above problems are not inherited in the analysis of discrete mechanical systems, e.g., molecular dynamics [8]. Unfortunately, on the available computational resources, only a very limited volume and time segment can be handled: the size of the observable body is at $10^{-6} \mathrm{~m}^{3}$ in volume, and the time segment is in $10^{-6} \mathrm{~s}$ scale. In these models, an ultra-high speed load is necessary. From experimental results, it is uncertain whether the mechanism of damage at ultra-high speed is similar to that at normal speed [9].

These drawbacks and open questions were the motivation, in the year 2000, when the first article of Silling was published, founding the basics of the peridynamic material model [10]; several publications have followed this.

This review aims to summarize the state of the art of the peridynamic theory of solids concisely, and highlight possible future directions. The review is concerned with the theoretical background, classification, and the recent applications of peridynamics. A deep investigation into the different approaches of the peridynamic modelling and the details of numerical implementation is outside the scope of this work. Based on the review, we are identifying and emphasizing four paths of developments in peridynamics: (1) handling materials nonlinearity with improvements in solver technology, (2) handling geometric nonlinearity with improved numerical stability, (3) efficiency of numerical solvers, and application of parallel computing, and (4) applications in coupled multiphysical problems.

The theory and classification of peridynamics are described in Section 1. The peridynamic specified enforcement of boundary conditions and the simulation of discontinuities are also covered in this section. Constitutive models of peridynamics and their applications are described in Section 2. All the references cited in this review can help to navigate and find the appropriate publication between the expansive set of peridynamics related papers.

\section{THE PERIDYNAMIC THEORY}

The name of the peridynamic model was proposed from ancient Greek words "near" and "force". The model is a strongly non-local model; the equilibrium (or the motion) of the material particle is affected by finite distance interactions [10].

With its first formulation during the motion and deformation, each particle pairs have an internal force between them, and the motion of a particle depends on the sum of these forces. Let $X$ and $X^{\prime}$ be two arbitrary particles of the body. Let $u(X, t)$ be the displacement field. The equations of motion of particle $X$ are:

$$
\begin{gathered}
\rho \cdot \ddot{u}=L_{u}+b, \\
L_{u}=\int_{V} f\left(u, u^{\prime}, t\right) d X^{\prime},
\end{gathered}
$$

where $b$ is a prescribed volumetric load, $\rho$ is the mass density and is the acceleration at particle $X$. The $L_{u}$ operator is the peridynamic force function. It represents the non-local effect on particle $X$ caused by the deformation of its environment.

\subsection{The Bond-Based Peridynamic (BB-PD) Model}

In Silling's pioneering article [10], the so-called bond based model was introduced, which proved to be very accurate. The peridynamic force, $f$ was the exclusive function of the relative displacement vector, $\eta=u^{\prime}-u$. The scheme of the bond-based kinematics and kinetics is shown in Fig. 1. Despite the simplicity of the model, it was proved to be highly successful at modelling crack initiation, propagation, and branching in brittle materials.

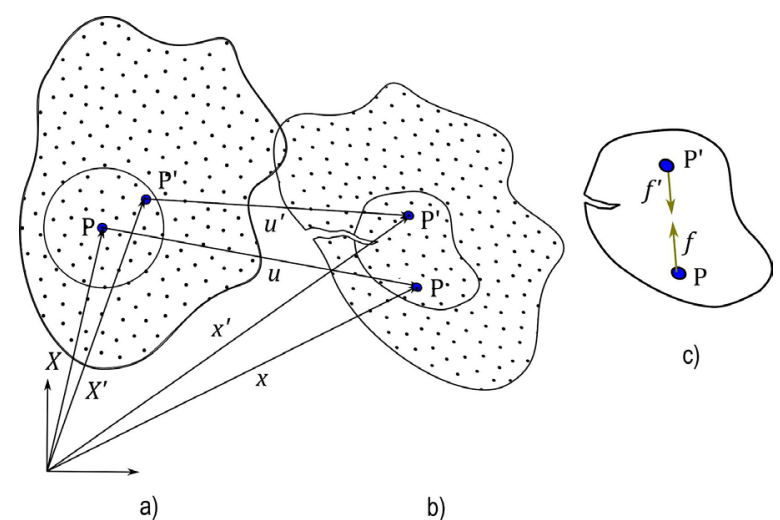

Fig. 1. The kinematics and kinetics of neighbours in Bond-based Peridynamic model; a) initial (undeformed) configuration of the body, b) current (deformed) configuration of the body,

c) the pairwise force between material points

In this model, the material was linear elastic originating from the relation between the stretch of the bond between the two particles and the linear peridynamic force. Nevertheless, the relation was strongly non-linear in a geometric sense. The direction of the peridynamic force was parallel with the current direction of the relative position.

In physical interactions, the intensity of the effects usually decreases rapidly with the distance between the interacting particles. It suggests defining 
a characteristic length to cut the radius of interaction around particle $X$. In peridynamic literature, this radius is referred to as material horizon, $\delta$. Let the reference vector of two arbitrary particles $X$ and $X^{\prime}$ be $\xi:=X-X^{\prime}$. The intensity of interaction between two points can be characterized by an influence function $\omega\left(X, X^{\prime}\right)$. The influence function is a strictly positive, finite support function. The radius of the support is the material horizon. When it depends only on the reference vector, $\omega\left(X, X^{\prime}\right)=\widehat{\omega}(\xi)$, it is called structureless. If it depends only on the Eulerian length of the reference vector, $\omega\left(X, X^{\prime}\right)=\breve{\omega}(|\xi|)$, it is called 'spherical'! [11].

Let $\mathcal{H}_{X}$ be the set of points in the body where $\omega\left(X, X^{\prime}\right)>0$. The $\mathcal{H}_{X}$ set is called to a family of particle $X$. Assuming a finite material horizon, Eq. (2) can be written in simplified form:

$$
L_{u}=\int_{\mathcal{H}_{X}} f\left(u, u^{\prime}, t\right) d X^{\prime} .
$$

In the peridynamic model, the force function $f$ contains all the constitutive information. In the previous two decades, this very simple model was applied in many fields of engineering: elasticity, plasticity, damage and fracture of solids, heat conduction and thermo-elasticity, etc. Some applications are summarized in Section 3.

Unfortunately, bond-based peridynamics have some disadvantages, e.g., the limited value of Poisson's ratio or the disability of the separation of volumetric from distortional deformation, which required the development of a more general theory of peridynamics.

\subsection{The State-Based Peridynamic (SB-PD) Mechanical Model}

Independently of the formulation in the above-cited articles, a generalized version of the peridynamic model was proposed by Silling et al. [12]. By employing the main concept of the state-based model, the peridynamic force between two material points is the function of the deformation state of the points.

The state of order $(m)$ is a tensor-vector function $\mathcal{A}\langle\cdot\rangle$. The most relevant applications of the states are the scalar $\left(0^{\text {th }}-\right.$ order $)$, vector $\left(1^{\text {st-order}}\right)$ and tensor (2nd-order) states.

The initial and the current configuration of a material point can be described by the reference state $\mathcal{X}$ and the deformed state $\mathcal{Y}$, respectively:

$$
\begin{gathered}
\mathcal{X}[X] \xi=\xi=X^{\prime}-X, \\
\mathcal{Y}[X] \xi=\xi+\eta=x^{\prime}-x .
\end{gathered}
$$

The displacement state $\mathcal{U}$ can be defined with a similar expression:

$$
\mathcal{U}[X] \xi=u^{\prime}-u .
$$

Neither the reference nor the deformed state, as shown in Fig, 2, have to be a continuous function of the vector variable.

However, a first-order state is a more general vector-vector function; therefore, the reduced form of the state can be defined. Vice versa, if a tensor is given, then an expanded state can be defined.

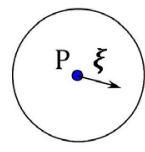

a)

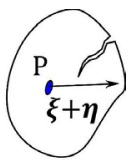

b)
Fig. 2. The neighbourhood (family) around a material point in State-based peridynamics; a) the reference state $\mathcal{X}[X]$, and b) the deformed state $\mathcal{Y}[X]$

Let $\omega\left(X, X^{\prime}\right)$ be the influence function. Let $\xi$ be the reference vector, and $W$ be a second-order tensor. The vector state expanded from the tensor is

$$
\mathcal{V}[X] \xi=W \cdot \xi .
$$

The tensor product of two vector states $A$ and $B$ is defined as:

$$
A^{*} B=\int_{\mathcal{H}_{X}} \omega\left(X, X^{\prime}\right) \cdot A\langle\xi\rangle \otimes B\langle\xi\rangle d X^{\prime} .
$$

The reference shape tensor is defined as

$$
K=\mathcal{X} * \mathcal{X} .
$$

Let $\mathcal{V}$ be a vector state. The tensor reduced form $W$ of the vector state is

$$
W=\left(\mathcal{V}^{*} \mathcal{X}\right) \cdot K^{-1},
$$

It is worth mentioning, assuming continuous displacements, the 0-limit of horizon leads to

$$
F(X)=\lim _{\delta \rightarrow 0}\left(\left(\mathcal{Y}^{*} \mathcal{X}\right) \cdot K^{-1}\right),
$$

where $F(X)$ is the deformation gradient of $\mathrm{CCM}$ at particle $X$ [12].

Let $a$ and $b$ are two vector states. The dot product between the two vector states is defined by

$$
a \bullet b=\int_{\mathcal{H}_{X}} A\langle\xi\rangle \cdot B\langle\xi\rangle d X^{\prime} .
$$

In CMM, the constitutive information is contained in the stress-strain relationship. In bondbased peridynamic theory, the material behaviour 
is described through the bond stretch-bond force relationship. In state-based peridynamics, the deformation state-force state relationship plays this role. The force state is a deformation-dependent vector-vector function. The equation of motion contains the force state.

$$
\begin{gathered}
\rho \cdot \ddot{u}=L_{u}+b, \\
L_{u}=\int_{\mathcal{H}_{X}} T[X, t]\langle\xi\rangle-T\left[X^{\prime}, t\right]\langle-\xi\rangle d X^{\prime} .
\end{gathered}
$$

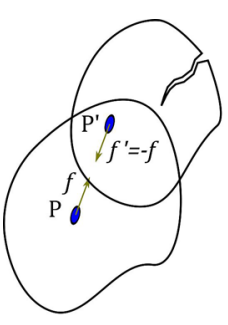

a)

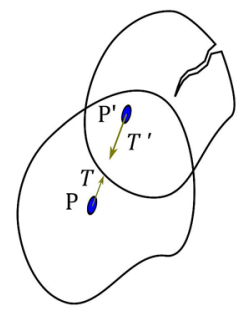

b)

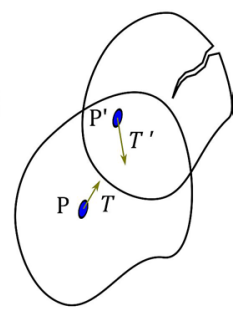

c)
Fig. 3. The kinetics of state-based peridynamics; a) properties of bond-based, b) ordinary- and c) non-ordinary statebased peridynamic force

It is worth introducing the reference and deformed direction states, respectively:

$$
\begin{gathered}
e_{r}\langle\mathcal{X}\rangle=\frac{\mathcal{X}}{|\mathcal{X}|}, \\
M[X]\left\langle X^{\prime}-X\right\rangle=\frac{\mathcal{Y}}{|\mathcal{Y}|} .
\end{gathered}
$$

In bond-based peridynamic theory, the bond force vector is parallel to the instant direction of the bond, and the magnitude is a pairwise function:

$$
f[X]\langle\xi\rangle=f\left(x, x^{\prime}\right) \cdot M=-f\left[X^{\prime}\right]\langle-\xi\rangle .
$$

In ordinary state-based peridynamic theory, the direction of the force vector state is parallel to the instant direction of the bond, but the magnitude of the force from $X$ to $X^{\prime}$ can be different from $X^{\prime}$ to $X$ :

$$
\begin{gathered}
T[X]\left\langle X^{\prime}-X\right\rangle=t\left(x, x^{\prime}\right) \cdot M, \\
f[X]\langle\xi\rangle=t\left(x, x^{\prime}\right) \cdot M-t\left(x^{\prime}, x\right) \cdot M^{\prime} .
\end{gathered}
$$

In non-ordinary state-based peridynamic theory, the direction of the force vector state is not parallel to the instant direction of the bond and the magnitude of the force from $X$ to $X^{\prime}$ can be different from $X^{\prime}$ to $X$ :

$$
\begin{gathered}
T[X]\left\langle X^{\prime}-X\right\rangle \neq t\left(x, x^{\prime}\right) \cdot M, \\
\left|T[X]\left\langle X^{\prime}-X\right\rangle\right| \neq\left|T\left[X^{\prime}\right]\left\langle X-X^{\prime}\right\rangle\right| .
\end{gathered}
$$

The relation of bond-based, ordinary state-based and the non-ordinary state-based peridynamic models are compared in Fig. 3.

Recently, other physical states (i.e., heat flux state, chemical concentration state, etc.) have been introduced and applied with success to model discontinuous field problems. In Section 3, some major fields of applications are shown related to the present concept.

\subsection{Damage Evolution and Crack Propagation}

In peridynamics, material damage is modelled by the bond failure between pairs of particles. Once a bond is broken, the interaction between particles provided by that bond will no longer exist during the rest of the analysis. The damage index $\varphi(X, t)$ has been suggested to measure the relation of damaged bonds and active bonds for any given particles.

$$
\varphi(X, t)=1-\frac{\int_{\mathcal{H}_{X}} \mu(\xi, t) d X^{\prime}}{\int_{\mathcal{H}_{X}} d X^{\prime}},
$$

and

$$
\mu(\xi, t)=\left\{\begin{array}{ll}
1 & \text { if the bond is active } \\
0 & \text { if the bond is broken }
\end{array} .\right.
$$

This is based on Eq. (23), $0 \leq \varphi(X, t) \leq 1$, where 0 represents the undamaged state and 1 represents the breakage of all the bonds of a given particle. The damage parameter $\mu(\xi, t)$ is used only to specify whether a bond is active or broken. The influence function $\omega\left(X, X^{\prime}\right)$ will equal to zero when $\mu(\xi, t)$ equals to zero.

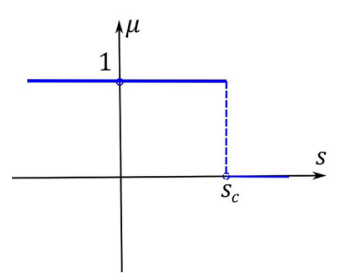

a)

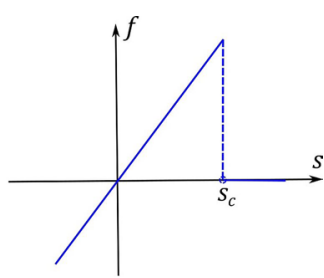

b)
Fig. 4. The damage model of peridynamics: a) The damage parameter-strain function, b) The force-strain function

This condition ensures that damaged bonds are excluded from the calculation of the equation of motion in the case of bond-based peridynamics, or they can be excluded during the calculation of deformation gradient tensor $F$ and shape tensor $K$ 
and that the resulting force state $T[X]<\xi>$ is set to 0 at damaged bonds.

For isotropic brittle materials, based on energy release rate equivalency of CCM and PD, Silling and Askari [13] derived the critical stretch for bond failure as

$$
s_{c}=\sqrt{\frac{5 \cdot G_{0}}{9 \cdot k \cdot \delta}},
$$

where, $G_{0}$ is the fracture energy of the material. The simplest damage parameter-strain and force-strain functions are shown in Fig. 4.

The disadvantage of the above, naive criteria is that, for some materials, it is an oversimplification. Conversely, using this approach, the complete PD fracture mechanics may be defined without the necessity of strain and stress concepts.

If the critical stretch for the failure of a given bond is dependent on conditions in all other bonds or the orientation of the bond, then a more sophisticated PD model is necessary. For anisotropic (composite) materials, Shang et al. [14] advised the introduction of multiple failure conditions, dependent on the end positions of the specified bond. In their article, the bond failure criteria correspond to mechanical stress components (calculated based on peridynamic force state) through the combination of "Maximum stress" and "Hashin" failure criteria of damage mechanics of composites.

If the energy release rate equivalency is too complicated or such traditional failure criteria cannot be adopted, then the critical stretch parameters can be obtained using experimental methods [15] and [16] or calibration using an inverse approach [17].

In concurrent methods (X-FEM, meshless methods), maintaining the crack geometry is a dedicated problem. To couple the chosen numerical solver with the level set method to follow the evolution of the crack is the most popular way to solve the above problem. This coupling is very robust (fast and stable), but the descriptions of crack initiation, branching or fragmentation are open research areas. Additionally, the crack-tip kinematics has to be controlled with artificial criteria [3]. Controversially, crack initiation, propagation (or complete fragmentation) in PD framework, using of any additional database on crack geometry is not necessary: peridynamic bond activity delivers the solution. Orientation and speed of the crack tip are also determined by the bond damage evolution. (e.g. [18] to [20])

In a paper by Zhao et al. [21], the crack propagation of double-notched and double-centre crack specimens were studied. Comparing X-FEM and peridynamics, PD is more computation consuming, and the numerical stability is an open question [22]. To improve the efficiency of $\mathrm{PD}$, additional efforts are usually necessary. The related results are summarized in Section 2.3.

In the detailed review by Diehl et al. [23], PD and experimental results were collected and compared based on 39 publications. The abilities of PD for wave propagation simulation were compared on four common experiments and on crack propagation problems was compared on five such experiments. Both the wave analysis and the crack geometry and velocity analysis of crack tip during dynamic loading show excellent accuracy with experimental results.

\subsection{Evaluation of Boundary Conditions}

In primal problems of classical continuum mechanics, mechanical problems are usually defined by a boundary value problem containing the governing partial differential equations, Eqs. (25) to (27), the essential, Eq. (28), and the natural, Eq. (29), boundary conditions (BC). These equations are valid only in the linearized theory of small displacements. The essential BCs are the constraints on a subregion of the body surface. The natural BCs are the surface tractions (or free surface) of the adjoint subregion of the body surface. The relation between the surface traction, the mechanical stress, and the partial derivatives of the displacement field are essential.

$$
\begin{gathered}
\rho \cdot \ddot{u}=\nabla \sigma+b, X \in V, \\
\sigma=\sigma(\varepsilon) \quad \text { or } \quad \dot{\sigma}=\dot{\sigma}(\varepsilon, \dot{\varepsilon}, \ldots), \\
\varepsilon=\frac{1}{2} \cdot(\nabla \otimes u+u \otimes \nabla), \\
u=u_{0}, \quad X \in S_{u}, \\
\sigma \cdot n=t, \quad X \in S_{t} .
\end{gathered}
$$

In peridynamic theory, the governing Eq. (1) is an integro-differential equation containing the time derivative and spatial integral of the displacement field. The direct evaluation of essential boundary condition in peridynamics is easily possible. It is worth mentioning that, during the numerical solution of peridynamic problems, to avoid the appearance of artificial bond failures near the essential boundary conditions, the failure mechanism has to be forbidden [13].

The evaluation of natural boundary conditions in a peridynamic framework is not trivial. Observing the theory of peridynamics, it is clear that a complete 
framework is built and that deformation, crack initiation, and propagation of a peridynamic body can be determined without the introduction and using the concept of spatial derivatives. The concept of surface traction is incompatible with the usual theory of peridynamics [10].

Considering the literature, three methods are developed to enforce natural boundary conditions: (1) in article of Le and Bobaru [24], using an (inner or artificial extern) boundary layer, the surface traction can be transformed to an artificial volumetric load. The thickness of the boundary layer is suggested as being equal to the peridynamic horizon. (2) By the articles [25] and [26], in correspondence (nonordinary) state-based peridynamics, using the approximation of strains, the stress tensor and the surface traction can be enforced directly. (3) Huang [27] recommends the modification of the equation of motion to take the local interactions into account to evaluate the surface traction. The first method is very popular in the numerical solution of bond-based and native (non-correspondence) problems. In problems of correspondence peridynamics, almost solely the second method can be found. The third method is very new and promising, although it has not been widely applied yet.

The enforcement of contact conditions between solids leads to strongly non-linear equations, because of the changing boundary conditions on the solid surfaces. In traditional computational methods (FEM, Meshless), depending on the magnitude of the displacements and the complexity of the behaviour (material, geometry, surface conditions), there are usual methods of discretization (from "node to node" to "surface to surface" discretization) and to find the contacting surface between loaded bodies (penalty, Lagrange multipliers and augmented Lagrange methods).

In peridynamics, similarly to particle methods, the surface of the body is not modelled. For modelling contact conditions, extraordinary efforts are necessary. In the recent literature, only a few articles deal with this problem. Most applications contain peridynamicrigid interactions inheriting contact models of particle methods. [28] The first dedicated article on this topic [29] deals with the contact of peridynamic and finite element models. An inverse isoparametric mapping technique and a node-to-surface contact algorithm are customized and implemented, and a penalty method enforcing displacement constraints is then incorporated for the transient analyses using the explicit time integration. Good agreement between numerical simulations and the analytical model is observed in the analysis of the residual velocities. The physical process of perforation is captured accurately in the simulations. In a recent paper [30], a new state-based (SB) non-local friction model was developed and compared with a bond-based (BB) contact model. The properties of various peridynamic contact models were demonstrated by applying them in finite element and meshfree peridynamic analyses of benchmark problems and an impact/penetration test. The BB model was computationally efficient and accurate to model Coulomb-friction but did not satisfy the physical principles of objectivity and balance of angular momentum. The SB model was physically correct and accurate to model Coulomb-friction, but the efficiency was less satisfactory.

\subsection{Concepts of Convergence}

Previously, the main concepts and definitions of the peridynamic material model were introduced. Some aspects of the relationship between the classical and peridynamic model were mentioned, but from the application point of view, one of the most important questions is the convergence abilities of the model.

The non-local nature of the peridynamic model allows the definition of the concept of convergence in different ways. On the one hand, it is worthwhile to observe the behaviour of the analytical solution of the peridynamic problem when the material horizon $\delta$ converges to zero. On the other hand, the convergence of the discretized model is also highly important.

The first aspect was studied in article [31] by Silling et al. This paper addresses the question of whether the peridynamic model for an elastic material reproduces the classical local model as this length scale approaches zero. If the motion, constitutive model, and any inhomogeneities are sufficiently smooth, then the peridynamic stress tensor converges to this limit to a Piola-Kirchhoff stress tensor that is a function only of the local deformation gradient tensor, similarly as in the classical theory. This limiting PiolaKirchhoff stress tensor field is differentiable, and its divergence represents the force density due to internal forces. The limiting, or collapsed, stress-strain model satisfies the conditions in the classical theory for angular momentum balance, isotropy, objectivity, and hyperelasticity, provided the original peridynamic constitutive model satisfies the appropriate conditions.

The convergence behaviour of peridynamics were studied in article [32] in which Mikata proved the $\delta$-convergence of elastic PD model to classical continuum model in case studies of statics (peristatics) and dynamics (peridynamics). He studied the wave 
dispersion abilities of the peridynamic model and, in the case of finite material horizon $\delta$, found an interesting difference to CCM: some peridynamic materials can have negative group velocities in certain regions of wavenumber.

The second numerical aspect was studied in articles [33] and [34]. Considering a one-dimensional (1D) peridynamic bar with an equidistant $\Delta x$ set of nodes, the region $[x-\delta, x+\delta]$ contains $2 m+1$ number of nodes, where $m=2 \delta \div \Delta x$ and $\div$ is the integer division operator. Based on this discretization, three types of convergency can be defined:

- The $m$-convergence: $\delta$ is fixed and $m \rightarrow \infty$. By the results of the article, the numerical peridynamic approximation converges to the exact non-local peridynamic solution for the given $\delta$.

- The $\delta$-convergence: $\delta \rightarrow 0$ and $m$ is fixed or increases but at a slower rate. (The number of nodes in the material horizon is fixed.) By the results, in this case, the numerical peridynamic approximation converges to an approximation of the classical solution almost everywhere. The larger $m$ is, the closer this approximation becomes. The convergence is not guaranteed to be uniform.

- The $(\delta m)$-convergence: $\delta \rightarrow 0$ and $m$ increases with decreasing $\delta$ with a higher rate than the $\delta$ goes to zero. In this case, we will see that the numerical peridynamic approximation converges to the analytical peridynamic solution and converges uniformly to the local classical solution, almost everywhere.

The results of standard numerical methods in fracture modelling usually suffer from mesh sensitivity. One of the main goals of the peridynamic model was to solve the problem of crack initiation propagation, branching, and material fragmentation without similar drawbacks.

Henke and Shanbhag [34] studied the effect of the spatial position of nodes on the results in the case of simple brittle crack propagation problems. They found that the case of a regular cubic set of nodes can cause artificial crack lines along the symmetry of the set. Using centroidal Voronoi tessellation (CVT) and an irregular set of nodes, this problem can be avoided. They mentioned that getting the same accuracy with these methods requires a higher number of nodes in the material horizon; $m$ has to be higher. Unfortunately, it increases the computational cost. Their work showed that the careful placement of nodes seems to eliminate any obvious signs of mesh sensitivity without incurring significant additional computational costs within the simulation. This is an important result because, in many cases, the added cost of generating point distributions is amortized over several simulation runs that share the same geometry. In their article, equivolumetric nodes were used, but it seems that when using improved methods of positioning and weighing peridynamic nodes, the drawbacks of mesh sensitivity and increasing computational cost can be neglected.

\section{CONSTITUTIVE MODELS AND APPLICATIONS IN PERIDYNAMICS}

Constitutive equations and the related material properties are adequately defined in the framework of CCM. These parameters originated in the strongly local nature of the CCM material model. The bondforce or the force state model is strongly non-local [35]; consequently, the material properties and their meanings are completely different. To find the relation between the two sets of properties, two methods were introduced.

In the native (or non-correspondence) methods, after analysing peridynamic kinematics and kinetics, deformation and force measures are defined. After the definition of the material model, its assumptions and the corresponding peridynamic material properties, deformation energy density is identified. Assuming equality between the classical material model and the peridynamic model through simple cases of deformation (homogenous deformation, uniaxial tension, pure shear), the relation between the material properties can be found.

In the correspondence methods, new material properties are not introduced. Instead, the peridynamic approximation of classical deformation and force measures are defined. It is demonstrated that the limits of these approximations are identical to the classical measures as the peridynamic material horizon approaches zero [31]. After the identification of the relationship between classical and peridynamic measures, the classical material properties can be used in peridynamic balance laws [31].

To demonstrate the two strategies, the derivation of equations of peridynamic elasticity is shown in the following subsection.

\subsection{Peridynamic Elasticity}

In the framework of bond-based peridynamics, Emmrich and Weckner [36] derived the relation between the displacement field and the peridynamic force vector. In their work, the strain $s$ and instant 
direction vector $e$ of the peridynamic bond was introduced as

$$
\begin{gathered}
s=\frac{\left|x^{\prime}-x\right|-\left|X^{\prime}-X\right|}{\left|X^{\prime}-X\right|}=\frac{\left|X^{\prime}+u^{\prime}-X-u\right|-\left|X^{\prime}-X\right|}{\left|X^{\prime}-X\right|}, \\
e=\frac{x^{\prime}-x}{\left|x^{\prime}-x\right|}=\frac{X^{\prime}+u^{\prime}-X-u}{\left|X^{\prime}+u^{\prime}-X-u\right|} .
\end{gathered}
$$

Assuming small deformations and linear elastic behaviour, one can introduce the pairwise potential in the deformed bond as a quadratic form of the instant relative position of the endpoints of the bond:

$$
w\left(X, X^{\prime}\right)=\frac{1}{2} \cdot\left(x^{\prime}-x\right)^{T} \cdot C\left(X, X^{\prime}\right) \cdot\left(x^{\prime}-x\right),
$$

where $C$ is the material stiffness tensor

$$
C=\frac{c}{|\xi|^{3}} \cdot(\xi) \otimes(\xi)
$$

Based on the equivalency of deformation energy of CCM and BB-PD material under simple deformation cases, the material property $c$ can be calculated as

$$
\begin{gathered}
c=\frac{18 \cdot k}{\pi \cdot \delta^{4}} \text { in three dimensional, } \\
c=\frac{72 \cdot k}{5 \cdot \pi \cdot \delta^{3}} \text { in two dimensional, } \\
c=\frac{18 \cdot k}{5 \cdot \pi \cdot \delta^{2}} \text { in one dimensional problems, }
\end{gathered}
$$

where $k$ is bulk modulus of the material.

The peridynamic pairwise force $f$ was written as the derivative of the elastic potential by the relative displacement:

$$
f=\frac{\partial w}{\partial\left(u^{\prime}-u\right)}=C \cdot\left(u^{\prime}-u\right) .
$$

One of the greatest drawbacks of the original bond-based elastic model is the limited value of Poisson's ratio [37]. Similar to Navier's central force elastic model, Poisson's ratio of the bond based peridynamic material is $v=1 / 4$ in spatial and $v=1 / 3$ in planar problems. In many practical cases, this limitation is acceptable (e.g., crack propagation of concrete structures [38] and [39]), but such an approximation is unacceptable in many engineering applications.

Despite the limited value of the Poisson's ratio, BB-PD has been applied with success in fracture mechanics of brittle materials [20], [38], [40], and other fields (see details in following subsections), from its beginning until recently. The original theory was analysed and compared with other fracture mechanics related methods, [18], [40] and [41], and it was modified to overcome the Poisson's ratio limitation [38] and [42] to [46].

As noted in Section 1.2, the shortcomings of BBPD led to the conception of the more generalized state-based peridynamics theory. The kinematics of deformation in the neighbour of a material point $X$ is written by the deformation state, $\mathcal{Y}$. In case of elastic behavior, the specific strain energy function depends on this quantity, $W=W(\mathcal{Y}, t)$. Similar to the previous paragraph of BB-PD, in case of small displacements, the force state $T$ is

$$
T=\mathbb{C} \cdot \mathcal{U} .
$$

The elements of the modulus state $\mathbb{C}$ can be written as second derivative of the strain energy destiny:

$$
\mathbb{C}=\left.\nabla_{\mathcal{Y}} \nabla_{\mathcal{Y}} W(\mathcal{Y})\right|_{\mathcal{Y}=\mathcal{X}} .
$$

According to Aguiar [47], relations between material properties of classical homogeneous isotropic linear elastic materials and elastic peridynamic materials are given more specifically. In the reference configuration, the reference direction $e_{r}<\mathcal{X}>$ and angular state $e_{n}\langle\mathcal{X}, \mathcal{X}\rangle$ can be defined, respectively as:

$$
\begin{gathered}
e_{r}\langle\mathcal{X}\rangle=\frac{\mathcal{X}}{|\mathcal{X}|}, \\
e_{n}\left\langle\mathcal{X}, \mathcal{X}^{\prime}\right\rangle=\frac{\left(1-e_{r}\langle\mathcal{X}\rangle \otimes e_{r}\langle\mathcal{X}\rangle\right) \cdot e_{r}\left\langle\mathcal{X}^{\prime}\right\rangle}{\sin (\alpha)},
\end{gathered}
$$

and $\alpha$ is the smallest angle between the two observed bonds $\mathcal{X}$ and $\mathcal{X}^{\prime}$.

Introducing the difference displacement ratio state by

$$
h=\frac{\mathcal{U}}{|\mathcal{X}|},
$$

the linear and shear strain states can be defined as

$$
\varepsilon=e_{r} \cdot h,
$$

$\gamma\left\langle\mathcal{X}, \mathcal{X}^{\prime}\right\rangle=\frac{1}{2} \cdot\left(e_{n}\left\langle\mathcal{X}, \mathcal{X}^{\prime}\right\rangle \cdot h\langle\mathcal{X}\rangle+e_{n}\left\langle\mathcal{X}^{\prime}, \mathcal{X}\right\rangle \cdot h\left\langle\mathcal{X}^{\prime}\right\rangle\right)$

Using these deformation states and reduced quadratic form of free energy function of an isotropic simple elastic material [48], yields 


$$
\begin{aligned}
W[h] & =\int_{\mathcal{H}_{X}} \int_{\mathcal{H}_{X}} \omega\left(\mathcal{X}^{\prime}, \mathcal{X}^{\prime \prime}\right) \cdot \\
& {\left[\begin{array}{c}
\frac{\alpha_{11}}{2}\left(\varepsilon^{\prime}\right)^{2}+\alpha_{12} \cdot \varepsilon^{\prime} \cdot \varepsilon^{\prime \prime}+ \\
+\frac{\alpha_{33}}{2}\left(\gamma^{\prime \prime \prime}\right)^{2}+\alpha_{13} \cdot \gamma^{\prime \prime \prime} \cdot \varepsilon^{\prime}
\end{array}\right] d X^{\prime \prime} d X^{\prime}, }
\end{aligned}
$$

where $\omega(\cdot, \cdot)$ is a given symmetric weight function and $\alpha_{11}, \alpha_{12}, \alpha_{13}$ and $\alpha_{33}$ are elastic peridynamic constants. Assuming equivalency between the elastic strain energy of classical Hookean material and the peridynamic elastic material, the relation of classical material properties and peridynamic material constants are

$$
\alpha_{11}=\frac{5 \cdot \mu}{m^{2}}, \alpha_{12}=\frac{9 \cdot \kappa-5 \cdot \mu}{2 \cdot m^{2}}, \alpha_{33}=\frac{20 \cdot \mu}{m^{2}},
$$

where the constant $m$ is defined by

$$
m=4 \cdot \pi \cdot \int_{0}^{\delta} \omega(\rho) \cdot \rho^{4} d \rho .
$$

The remaining constant $\alpha_{13}$ is the coupling constant in elastic strain energy between linear and angular strains. A similar material property is not available in classical elasticity. Assuming a finite peridynamic horizon if the quadratic free energy function of the material is given, then the value of $\alpha_{13}$ can be determined from Eq. (45). In this sense, the material property $\alpha_{13}$ means a correction to compensate the effect of finite peridynamic horizon.

An alternative method to determine the statebased peridynamic material constants is to decompose the deformation state to dilatation and deviatoric terms. Silling et al. [12] and Madenci and Oterkus [49] followed slightly different ways during this decomposition and achieved different results.

In Silling's work the force state is

$$
\begin{aligned}
T[X]\langle\xi\rangle & =M[X]\langle\xi\rangle \cdot \\
& \cdot\left(\frac{3 \cdot k}{m} \cdot \omega \cdot \mathcal{X} \cdot \theta+\frac{15 \cdot \mu}{m} \cdot \omega \cdot e^{d}\right),
\end{aligned}
$$

where $k$ and $\mu$ are the bulk and shear modulus, respectively. The scalar reference and extension state are defined, respectively:

$$
\begin{gathered}
\mathcal{X}=|\mathcal{X}|, \\
e(\mathcal{Y})=|\mathcal{Y}|-|\mathcal{X}| .
\end{gathered}
$$

Weighted volume has to be introduced:

$$
m=(\omega \cdot \mathcal{X}) \bullet \mathcal{X} .
$$

The dilatation- and deviatoric extension states are defined over the scalar extension state as:

$$
\begin{aligned}
& \theta(\mathcal{Y})=\frac{3}{m} \cdot(\omega \cdot \mathcal{X}) \bullet e(\mathcal{Y}), \\
& e^{d}(\mathcal{Y})=e(\mathcal{Y})-\frac{\theta(\mathcal{Y}) \cdot|\mathcal{X}|}{3} .
\end{aligned}
$$

In Madenci's work

$$
\begin{aligned}
T[X]\langle\xi\rangle & =2 \cdot \delta \cdot M[X]\langle\xi\rangle \cdot \\
& \cdot\left(a \cdot \frac{\mathcal{Y} \cdot \mathcal{X}}{|\mathcal{Y}| \cdot|\mathcal{X}|} \cdot \theta+b \cdot \frac{|\mathcal{Y}|-|\mathcal{X}|}{|\mathcal{X}|}\right) .
\end{aligned}
$$

The dilatation-state in this context was:

$$
\theta(\mathcal{Y})=d \cdot \delta \cdot \int_{\mathcal{H}_{X}} \frac{|\mathcal{Y}|-|\mathcal{X}|}{|\mathcal{X}|} \cdot \frac{\mathcal{Y} \cdot \mathcal{X}}{|\mathcal{Y}| \cdot|\mathcal{X}|} d X^{\prime}
$$

The constants $a, b$ and $d$ in the above expressions were:

$$
a=\frac{3 \cdot k-5 \cdot \mu}{2}, \quad b=\frac{15 \cdot \mu}{2 \cdot \pi \cdot \delta^{5}}, \quad d=\frac{9}{4 \cdot \pi \cdot \delta^{4}} .
$$

In articles and book by Madenci and Oterkus [49], and [50], the elastic peridynamic material properties were also analysed. In their works, the peridynamic states defined above were applied on a set of discrete material points.

Van Le and Bobaru [51] showed that the applicability of the above results is limited. Madenci's model is valid only in the case of small strains and infinitesimal rotations; Silling's model is valid in case of small strains and finite rotations [51]; Van Le has not found a valid peridynamic model for the case of finite strain and finite rotation deformations.

Using the approach of the above methods, in the applications, the concepts of strain and stress are not necessary. In the literature, this type of approach is referred to as a native or non-correspondence peridynamic model.

As mentioned, with the second method, the introduction of peridynamic material properties is not necessary. The peridynamic approximation of the displacement gradient can be written as

$$
\nabla u=\left(\mathcal{U}^{*} \mathcal{X}\right) \cdot K^{-1} .
$$

The approximating engineering strain tensor and the stress tensor are

$$
\begin{gathered}
\varepsilon=\frac{1}{2} \cdot\left(\mathcal{U}^{*} \mathcal{X}+\mathcal{X} * \mathcal{U}\right) \cdot K^{-1}, \\
\sigma=C \cdot \varepsilon
\end{gathered}
$$


Using Eq. (7) of expanded vector state, the peridynamic force state is:

$$
T[X]\langle\xi\rangle=\omega(|\xi|) \cdot \sigma \cdot K^{-1} \cdot \xi .
$$

Substituting Eq. (60) into the equation of motion, Eq. (13):

$$
\begin{aligned}
\rho \cdot \ddot{u} & =\int_{\mathcal{H}_{X}} \omega(|\xi|) \cdot \sigma^{\prime} \cdot K^{\prime-1} \cdot \xi^{\prime} d X^{\prime}- \\
& -\int_{\mathcal{H}_{X}} \omega(|\xi|) \cdot \sigma \cdot K^{-1} \cdot \xi d X^{\prime}+b(X) .
\end{aligned}
$$

The correspondence peridynamic model has the ability to describe large displacement, rotation, and deformation problems - theoretically. In numerical applications, some stability issues of this method were reported. Recently, several stabilization methods have been suggested for the original correspondence model [22] and [52] to [54], but this area still has some open questions and challenges.

Parallel to the development of a peridynamic model for three-dimensional elastic bodies, the research of other special fields of elasticity appeared in the peridynamic literature. Silling and Bobaru [55], used peridynamics to model the behaviour of rubberlike membranes and fibres. Gerstle et al. [38] introduced the micropolar bond-based peridynamics and used it to solve plane stress problems of concrete structures. With the micropolar model, one can avoid the drawback of fixed Poisson's ratio anomaly of bondbased peridynamics. Kilic et al. [56] published the first article about the peridynamics of composite laminates. They modelled the effect of fibre orientation on the crack propagation in planar composites. Oterkus et al. proved the validity of peridynamic theory through qualitative and quantitative comparisons against the test results for a stiffened composite curved panel with a central slot under combined internal pressure and axial tension [15]. The crack propagation of composite laminates under dynamic (impact and explosive) loads was modelled by peridynamics [19] and [57]. The peridynamic predictions correlated well with the experimental results available in the literature. Crack propagation in ceramic matrix composites [58] and in fibre-reinforced composites [59] was modelled using the peridynamic framework.

State-based peridynamics of beams was developed [60] to solve the problem of the Eulerbeam. Yang et al. formulated the peridynamic theory of functional graded Thimosenko-beam [61]. The validation of the model was proven by testing different boundary conditions and loads. The results showed good agreement with analytical and finite element results.

\subsection{The State-Based Peridynamic Plasticity}

Although plasticity can be included in the bondbased theory by permitting permanent deformation of individual bonds [62], this results in permanent deformation of a material undergoing volumetric strain (without shear). Metal forming experiments have suggested volumetric incompressibility of plastic deformation. It means that only shear deformations can induce plastic response [63]. Consequently, the bond based peridynamic model, in its original form, cannot describe the plastic deformation of metals.

In the work of Mitchell [64] and Littlewood et al. [65], the ordinary state-based (non-correspondence) peridynamics was used to model elasto-plasticity and viscoplastic behaviour. The first paper is a theoretical foundation of PD plasticity without qualitative or quantitative results. The second publication contains a quantitative comparison between PD and experimental results.

In article of Mitchell [64], PD plasticity was defined over continuous sets of points, using integral forms and Frechet-derivatives. In the article of Madenci and Oterkus, a discretized set of points was used to introduce inelasticity [50].

Plastic deformation, in the case of most metals, is independent of volumetric deformation and hydrostatic pressure. To separate the hydrostatic effects from shear effects, the decomposition of deformation state is required to volumetric and deviatoric part. Using Eqs. (52) and (53), the deformation state can be decomposed to dilatation $\theta$ and deviatoric $e^{d}$ terms. Using this assumption, the plastic deformation depends only on the second term of Eq. (48). The deviatoric part of the deformation extension state can be decomposed to elastic $e^{d e}$ and plastic $e^{d p}$ parts:

$$
e^{d}=e^{d e}+e^{d p} .
$$

Hence, the rate form of the balance Eq. (48) is

$$
\dot{t}=\frac{3 \cdot k}{m} \cdot \omega \cdot \mathcal{X} \cdot \dot{\theta}+\frac{15 \cdot \mu}{m} \cdot \omega \cdot\left(\dot{e}^{d}-\dot{e}^{d p}\right) .
$$

Following the way of classical plasticity [66], a perfectly plastic flow rule, consistency parameter $(\lambda)$ and Kuhn-Tucker conditions were derived. Based on these quantities and relations, through linearization of the deformation and force states, the implicit time integration of the plastic model was developed.

The peridynamic yield function $f$ of perfectly plastic material is

$$
f\left(t^{d}\right)=\psi\left(t^{d}\right)-\psi_{0} \leq 0 .
$$


The relationship between the peridynamic yield condition $\psi_{0}$ and yield stress of the classical material model is

$$
\psi_{0}=\frac{75}{8 \cdot \pi} \cdot \frac{E_{y}^{2}}{\delta^{5}},
$$
where $E_{y}$ is usually measured in uniaxial $E_{y}=\frac{\sigma_{1}}{\sqrt{3}}$ or
pure shear $E_{y}=\sigma_{12}$ stress conditions.

Later, in the article [50], peridynamic plastic deformation according to the von Mises yield criterion with isotropic hardening was also developed.

Stefanelli et al. [67], in the framework of nonlocal vector calculus, analysed the solution of the peridynamic elasto-plastic problem. The existence and convergence to the CCM solution were proved. Based on this and other non-local vector calculus (NLVC) related publications, one can assume that the NLVC could be a pillar of the generalized, robust mathematical background behind the peridynamic theory.

The correspondence peridynamic model was first applied on viscoplasticity by Foster et al. [68]. Littewood reported a plastic localization model using peridynamics [65]. Sandia National Laboratories announce "Sandia Fracture Challenges", in every second year, with more and more challenging problems to predict material damage and crack propagation in ductile materials. State-based peridynamic solutions are often submitted, the results are published. and the accuracy of SB-PD is improving, but acceptable [69] to [71].

\subsection{Relation of Peridynamics with Classical and Experimental Results}

Out of the nearly 500 referred articles of peridynamics published in the previous two decades, 40 are related to damage, 124 are about fracture, and 70 are crackrelated articles. On some fields of applications, only a few papers were published, although other fields were more fruitful (Table 1).

Topics with few publications are those for which the authors mostly only develop the PD framework of the specified problem and prove the capability of peridynamics to simulate the phenomena on some essential problems. On the more studied areas, usually the formulation of PD is better established and (next to the essential problems) more real world applications can be found. Here we would like to highlight only a few application areas where peridynamics have been successfully applied.

The most frequent research area in peridynamics is fracture mechanics. In applications, the comparability and relation between the classical and the PD material models are essential. The relation between peridynamics and material properties of classical fracture mechanics has been widely studied. The peridynamic non-local J-integral has also been thoroughly analysed in [72] and [24]. The displacement-based J-integral formulation, developed in [73], can be obtained with bond-based or state-based peridynamic models. In the article of Panchadhara and Gordon [74], a new peridynamic stress intensity factor (SIF) was introduced. This quantity enables peridynamics an effective way of replacing the more or less ad-hoc fracture criteria to motivate fracture initiation and direction of fracture propagation in conventional numerical techniques of fracture mechanics. It was proved that the peridynamic SIF used in conventional numerical methods improves the robustness of the solution. Furthermore, peridynamic SIFs may be applied to study complex fracture physics, such as the branching and interaction of stress waves with propagating fractures that would otherwise be difficult using conventional numerical methods.

Table 1. Number of papers on the fields of PD applications

\begin{tabular}{lc}
\hline Field of application & Number of published papers \\
\hline Elasticity & 23 \\
\hline Heat conduction, Thermoelasticity & 12 \\
\hline Fluid transport and porose material & 1 \\
\hline Plasticity & 15 \\
\hline Damage & 10 \\
\hline Fracture & 76 \\
\hline Fatigue & 9 \\
\hline Structural Stability & 1 \\
\hline Corrosion and chemical damage & 4 \\
\hline Electricity & 4 \\
\hline Multiscale problems & 2 \\
\hline Phase transformation & 2 \\
\hline Technology and manufacturing & 4 \\
\hline
\end{tabular}

The most frequent research area in peridynamics is fracture mechanics. In applications, the comparability and relation between the classical and the PD material models are essential. The relation between peridynamics and material properties of classical fracture mechanics has been widely studied. The peridynamic non-local J-integral has also been thoroughly analysed in [72] and [24]. The displacement-based J-integral formulation, developed in [73], can be obtained with bond-based or state-based peridynamic models. In the article of Panchadhara and Gordon [74], a new peridynamic stress intensity 
factor (SIF) was introduced. This quantity enables peridynamics an effective way of replacing the more or less ad-hoc fracture criteria to motivate fracture initiation and direction of fracture propagation in conventional numerical techniques of fracture mechanics. It was proved that the peridynamic SIF used in conventional numerical methods improves the robustness of the solution. Furthermore, peridynamic SIFs may be applied to study complex fracture physics, such as the branching and interaction of stress waves with propagating fractures that would otherwise be difficult using conventional numerical methods.

The linear elastic and later hyperelastic extension models were applied to describe the crack propagation phenomena in homogenous and heterogenic bodies of 3-D or 2-D (plane stress/strain) geometries [15] and [75]. The problem of tearing and crack propagating in shells and fibres were also solved with promising qualitative results [55]. These papers demonstrated the ability of PD in this field through different problems with visualized results but did not contain quantitative data comparable with the results of classical analytic or numerical methods.

O'Grady and Foster published the NOSBPD model of a Kirchoff-Love plate. [37] In their paper, the simple bent square plates with different boundary conditions were studied and compared with analytical solutions. The $\delta$-convergence of transverse displacements of the applied model was shown. A demonstration of brittle fracture in a bending (double torsion) plate showed the potential of this model to broaden the class of problems that can realistically be modelled using peridynamics.

Chowdhury et al. derived the PD formulation of thin and thick curved plates under the assumptions of linear elasticity. [76] Their proposal on the peridynamic shell theory is numerically assessed against simulations on static deformation of spherical and cylindrical shells, that of flat plates and quasistatic fracture propagation in a cylindrical shell.

Recently, based on a more general SB-PD model, the complete Reissner-Mindlin shell theory was adapted to the peridynamic framework, and validity was proven through several numerical problems of continuous and damaged, discontinuous thick shells [77]. The tested models (containing attractively low PD nodes) were compared to the analytical and FEM results and showed excellent accuracy in the cases of small and large displacements/deformations.

Crack initiation, propagation, and bifurcation were modelled in the framework of PD in multilayer structures [78] and composite laminates [56], [19], [57] and [79] under thermal and dynamic loads. Mechanical damage was modelled in impact loaded layered glass [80], tensioned hydrated cement paste [81] and glasspolycarbonate-glass layered structure [59] by BB-PD. Also, a modified BB-PD was used to model dynamic fracture in functionally graded materials [82]. The results of PD models were compared with different experimental setups. The capability of peridynamics to simulate different damage modes of multilayer and composite structures were proven. The main advantage of PD against other existing models is the ability to identify all of the failure modes without simplifying assumptions. Damage is inherently calculated in a PD analysis without special procedures, making progressive failure analysis more practical. The price for this completeness is the necessity of severe PD nodes (each layer of composite has its own set of nodes and bonds are defined in plane and in transverse directions) and the time-consuming computations.

The BB-PD model of heat transfer was introduced by Bobaru and Duangpanya [83]. Using the concept of very simple and intuitive thermal-bond, 1-D transient heat conduction was simulated under different boundary conditions. - and -convergence of the model were observed. Both converged to the analytic solutions.

Fully coupled OSB-PD thermo-elasticity was developed by Oterkus et al. [84]. The derivation of the governing equations was based upon thermodynamic considerations. The capability of a PD model was tested and proved on simple 1-D and 3-D convergence studies to analytic, FEM and BEM results. In their paper, practical problems were not studied.

Thermo-mechanically induced damage and fracture in viscoelastic solids was simulated using OSB-PD by Madenci and Oterkus. The deformation analysis successfully captured the relaxation behaviour of the material. The numerical results concerned first the verification problems and subsequently, a doublelap joint with a viscoelastic adhesive where failure nucleates and grows.

The very complex thermo-plastic material behaviour was simulated in the framework of NOSB-PD by Amani et al. [86]. The Johnson-Cook constitutive and damage models were used. Two experimental configurations (Taylor-bar impact test (TBIT) and Kalthoff-Winkler test (KWT)) were simulated and compared. The numerical results agreed well with the available experimental data, instead of the moderate number of peridynamic nodes (5400 in TBIT and 60291 in KWT).

Recently, thermo-mechanically induced fracture was also analysed in peridynamics [87]. This paper 
presented an effective way to use a variable grid size in a weakly coupled thermo-mechanical peridynamic model. The proposed numerical method was equipped with a stretch control criterion to transform the grid discretization adaptively. Hence, the fine grid spacing was only used in limited zones where it was required. The computation time using the adaptive method was less than one third of the original fine grid. Predicting complex crack patterns without any a priori hypothesis on cracks is automatic in this model.

\section{CONCLUSIONS}

Aiming to highlight findings that are concerned with theory and applications of a new, strongly non-local method of continuum mechanics - peridynamics foundations of the method are reviewed while seeking new paths in future developments.

In peridynamics, the advantages of classical continuum mechanics and molecular dynamics are alloyed (especially in the field of fracture mechanics) based on the theory and work of Silling. It was found that the nonlocality of peridynamics plays a significant role in the ability to simulate crack propagation, branching, and coalescence in brittle materials. Peridynamics was shown to describe the above phenomena without any artificial conditions of crack-tip velocity with excellent accuracy to experimental data in plenty of standardized and "realworld" configurations. Using the overview of the works published in the recent decades, directions for future developments are identified in four paths:

(1) Applying peridynamics in non-linear and ductile fracture mechanics possess broad potential. Combining peridynamics and ductility is challenging, and the usual explicit solvers of peridynamics are not sufficiently effective. The development of implicit and/or more effective explicit solvers is needed.

(2) Numerical stability issues were observed in large deformation peridynamics, and only a few authors have dedicated their work to these problems. The relation between the meshless methods and the peridynamics is established. Based on this fact, the introduction of higher-order peridynamics may provide a solution to these stability issues.

(3) Analysis of numerical stability, robustness, and efficiency of peridynamics are also research directions that may require more focus in the coming years. The peridynamics are very accurate, but the speed and efficiency in "industrial problems" are lower than the concurrent methods, such as Extended-FEM or meshless methods. Coupling of PD with these methods is very efficient [16] and [88] to [90] and, as an alternative solution the parallel computing (e.g. supercomputers or GPGPU), seems natively applicable in peridynamics, which could increase the speed and efficiency of the method [91] to [93].

(4) It has also been noticed that there is an emerging number of coupled multiphysical and multidisciplinary works. In the case of successful implementation of peridynamics on mechanics of large deformation of solids, the research of separation (crack propagation) or merging of soft tissue would initiate and open opportunities for further biomechanical and other biophysical applications.

\section{ACKNOWLEDGEMENTS}

The authors would like to thank the financial support for publication by Project no. 2020-4.1.1TKP2020, which has been implemented with the support provided by the Ministry of Innovation and Technology of Hungary from the National Research, Development and Innovation Fund, financed under the TKP 2020 funding scheme.

\section{REFERENCES}

[1] Milne, I., Ritchie, R. O., Karihaloo, B. (2007). Comprehensive Structural Integrity, Elsevier Science, New York.

[2] William T. B.; Roch J. S. (2010). ASM Handbook - Failure Analysis and Prevention, vol. 11, ASM International, New York.

[3] Sun, C. T., Jin Z. H. (2012). Fracture Mechanics, Elsevier, Berlin.

[4] Lemaitre, J., Desmorat, R. (2005). Engineering Damage Mechanics, Springer, Berlin.

[5] Baaser, H., Gross, D. (2003). Remarks on the use of continuum damage models and on the limitations of their applicability in ductile fracture mechanics. Baaser, H., Hutter, K. (eds.), Deformation and Failure in Metallic Materials, Springer, Berlin, p. 345-362, D0l:10.1007/978-3-540-36564-8_14.

[6] Khoei, A. R. (2015). Extended Finite Element Method: Theory and Applications, Wiley, Oxford, DOl:10.1002/9781118869673.

[7] Chen, Y., Lee, J., Eskandarian, A. (2006). Meshless Methods in Solid Mechanics, Springer, Berlin.

[8] Huang, D., Wang, M., Lu, G. (2014). Continuum fracture analysis and molecular dynamic study on crack initiation and propagation in nanofilms. Journal of Nano-materials, vol. 1, p. 1-7, DOI:10.1155/2014/732434.

[9] Lee, J. G. (2012). Computational Material Science, CRC Press, New York.

[10] Silling, S. A. (2000). Reformulation of elasticity theory for discontinuities and long range forces. Journal of Mechanics 
and Physics of Solids, vol. 48, no. 1, p. 175-209, D0l:10.1016/ S0022-5096(99)00029-0.

[11] Silling, S. A. (2016). Introduction to peridynamics. Bobaru, F., Foster, J. T., Geubelle, S., Silling, S. A. (Eds.) Handbook of Peridynamic Modeling, Chapman and Hall/CRC, New York, p. 63-98.

[12] Silling, S. A., Epton, M., Weckner, O., Xu, J., Askari, E. (2007). Peridynamic States and Constitutive Modeling. Journal of Elasticity, vol. 88, p. 151-184, D0l:10.1007/s10659-0079125-1.

[13] Silling, S. A., Askari, E. (2005). A meshfree method based on the peridynamic model of solid mechanics. Computers and Structures, vol. 83, no. 17-18, p. 1526-1535, D0l:10.1016/j. compstruc.2004.11.026.

[14] Shang, S., Qin, X., Li, H., Cao, X. (2019). An application of nonordinary state-based peridynamics theory in cutting process modelling of unidirectional carbon fiber reinforced polymer material. Composite Structures, vol. 226, D0l:10.1016/j. compstruct.2019.111194.

[15] Oterkus, E., Madenci, E. (2012). Peridynamic analysis of fiber-reinforced composite materials. Journal of Mechanics of Materials and Structures, vol. 7, p. 45-84, D0l:10.2140/ jomms.2012.7.45.

[16] Oterkus, E., Madenci, E., Weckner, O., Silling, S. A., Bogert, P., Tessler, A. (2012). Combined finite element and peridynamic analyses for predicting failure in a stiffened composite curved panel with a central slot. Composite Structures, vol. 94, no. 3, p. 839-850, D0l:10.1016/j.compstruct.2011.07.019.

[17] Colavito, K., Kilic, B., Celik, E., Madenci, E., Askari E., Silling, S. A. (2007). Effect of void content on stiffness and strength of composites by peridynamic analysis and static indentation test. Proceedings of the 48th AIAA/ASME/ASCE/AHS/ASC Structures, Structural Dynamics and Materials Conference, $\mathrm{p}$. 2007-2021, DOI:10.2514/6.2007-2251.

[18] Bobaru F., Zhang, G. (2015). Why do cracks branch? A peridynamic investigation of dynamic brittle fracture. International Journal of Fracture, vol. 196, no. 1, p. 59-98, D0l:10.1007/s10704-015-0056-8.

[19] Chaoyang, S., Zaixing, H. (2016). Peridynamic simulation to impacting damage in composite laminate. Composite Structures, vol. 138, p. 335-341, D0l:10.1016/j. compstruct.2015.12.001.

[20] Wang, Q., Wang, Y., Zan, Y., Lu, W., Bai, X., Guo, J. (2018). Peridynamics simulation of the fragmentation of ice cover by blast loads of an underwater explosion. Journal of Marine Science and Technology, vol. 23, no. 1, p. 52-66, D0l:10.1007/ s00773-017-0454-x.

[21] Zhao, J., Tang, H.-S. Yue, S. (2017). Peridynamics versus XFEM: a comparative study for quasistatic crack problems. Frontiers of Structural and Civil Engineering, vol. 12, D0l:10.1007/ s11709-017-0434-6.

[22] Silling, S. A. (2017). Stability of peridynamic correspondence material models and their particle discretizations. Computer Methods in Applied Mechanics and Engineering, vol. 322, p. 42-57, D0l:10.1016/j.cma.2017.03.043.

[23] Diehl, P., Prudhomme, S., Levesque, M. (2019). A Review of Benchmark Experiments for the Validation. Journal of Peridynamic and Non-local Models, vol. 1, p. 14-35, DOI:10.1007/s42102-018-0004-X.

[24] Le, Q. V., F. Bobaru, F. (2018). Surface corrections for peridynamic models in elasticity and fracture. Computational Mechanics, vol. 61, no. 4, p. 499-518, D0l:10.1007/s00466017-1469-1.

[25] Warren, T. L., Silling, S. A., Askari, A., Weckner, O., Epton, M. A., $\mathrm{Xu}, \mathrm{J}$. (2009). A non-ordinary state-based peridynamic method to model solid material deformation and fracture. International Journal of Solids and Structures, vol. 46, no. 5, p. 1186-1195, DOI:10.1016/j.jjsolstr.2008.10.029.

[26] Wu, C. T., Ren, B. (2015). A stabilized non-ordinary statebased peridynamics for the ductile material failure analysis in metal machining process. Computer Methods in Applied Mechanics and Engineering, vol. 291, p. 1-40, D0l:10.1016/j. cma.2015.03.003.

[27] Huang, Z. (2019). Revisiting the peridynamic motion equation due to characterization of boundary conditions. Acta Mechanica Sinica, vol. 35, no. 5, p. 972-980, D0l:10.1007/ s10409-019-00860-3.

[28] Littlewood, D. (2010). Simulation of Dynamic Fracture Using Peridynamics, Finite Element Modeling, and Contact. ASME International Mechanical Engineering Congress and Exposition, Proceedings (IMECE), vol. 9, p. 209-217, D0I:10.1115/IMECE2010-40621.

[29] Lee, J., Liu, W., Hong, J.-W. (2016). Impact fracture analysis enhanced by contact of peridynamic and finite element formulations. International Journal of Impact Engineering, vol. 87, p. 108-119, D0l:10.1016/j.ijimpeng.2015.06.012.

[30] Kamensky, D., Behzadinasab, M., Foster, J. T., Bazilevs, Y. (2019). Peridynamic modeling of frictional contact. Journal of Peridynamics and Non-local Modeling, vol. 1, p. 107-121, DOI:10.1007/s42102-019-00012-y.

[31] Silling, S. A., Lehoucq, R. B. (2008). Convergence of peridynamics to classical elasticity. Journal of Elasticity, vol. 93, p. 13-37, D0l:10.1007/s10659-008-9163-3.

[32] Mikata, Y. (2012). Analytical solutions of peristatic and peridynamic problems for a $1 \mathrm{D}$ infinite rod. International Journal of Solids and Structures, vol. 49, p. 2887-2897, DOI:10.1016/j.ijsolstr.2012.02.012.

[33] Bobaru, F., Yang, M., Alves, L. F., Silling, S. A., Askari, E., Xu, J. (2009). Convergence, adaptive refinement, and scaling in 1D peridynamics. International Journal for Numerical Methods in Engineering, vol. 77, p. 852-877, D0l:10.1002/nme.2439.

[34] Henke, S. F., Shanbhag, S. (2014). Mesh sensitivity in peridynamic simulations. Computer Physics Communications, vol. 185, pp. 181-193, D0l:10.1016/j.cpc.2013.09.010.

[35] Eringen, A. C. (2002). Non-local Continuum Field Theories, Springer-Verlag New York Inc., New York.

[36] Emmrich, E. and Weckner, 0. (2006). The peridynamic equation of motion in non-local elasticity theory. III. European Conference on Computational Mechanics, p. 1-8, DOI:10.1007/1-4020-5370-3_62.

[37] O'Grady, J., Foster, J. T. (2014). Peridynamic plates and flat shells: A non-ordinary, state-based model. International Journal of Solids and Structures, vol. 51, no. 25-26, p. 45724579, D0I:10.1016/j.jjsolstr.2014.09.003. 
[38] Gerstle, W., Sau, N., Silling, S. A. (2007). Peridynamic modeling of concrete structures. Nuclear Engineering and Design, vol. 237, no. 12-13, p. 1250-1258, D0l:10.1016/j. nucengdes.2006.10.002.

[39] Huang, D., Zhang, Q., Qiao, P. Z. (2011). Damage and progressive failure of concrete structures using. Science China Technological Sciences, vol. 54, no. 3, p. 591-596, D0I:10.1007/s11431-011-4306-3.

[40] Agwai, A. Guven, I., Madenci, E. (2011). Predicting crack propagation with peridynamics: a comparative study. International Journal of Fracture, vol. 171, no. 1, p. 65-78, DOI:10.1007/s10704-011-9628-4.

[41] Weckner, O., Brunk, G., Epton, M. A., Silling, S. A. Askari, E. (2009). Comparison between local elasticity and non-local peridynamics.

[42] Dan, H., Guangda, L. Pizhong, Q. (2015). An improved peridynamic approach for quasi-static elastic deformation and brittle fracture analysis. International Journal of Mechanical Sciences, vol. 94-95, p. 111-122, D0l:10.1016/j. ijmecsci.2015.02.018.

[43] Zhu, Q.-Z., Ni, T. (2017). Peridynamic formulations enriched with bond rotation effects. International Journal of Engineering Science, vol. 121, p. 118-129, D0l:10.1016/j. ijengsci.2017.09.004.

[44] Liu, W., Hong, J.-W. (2012). Discretized peridynamics for linear elastic solids. Computational Mechanics, vol. 50, no. 5, p. 579-590, DOI:10.1007/s00466-012-0690-1.

[45] Wang, Y., Zhou, X., Wang, Y., Shou, Y. (2018). A 3-D conjugated bond-pair-based peridynamic formulation for initiation and propagation of cracks in brittle solids. International Journal of Solids and Structures, vol. 134, p. 89-115, D0l:10.1016/j. ijsolstr.2017.10.022.

[46] Diana, V., Casolo, S. (2019). A full orthotropic micropolar peridynamic formulation for linearly elastic solids. International Journal of Mechanical Sciences, vol. 160, p. 140-155, D0l:10.1016/j.ijmecsci.2019.06.036.

[47] Aguiar, A. R. (2016). On the determination of a peridynamic constant in a linear constitutive model. Journal of Elasticity, vol. 122, p. 27-39, D0l:10.1007/s10659-015-9531-8.

[48] Aguiar, A. R., Fosdick, R. L. (2014). A constitutive model for a linearly elastic peridynamic body. Mathematics and Mechanics of Solids, vol. 19, p. 502-523, DOI:10.1177/1081286512472092.

[49] Madenci, E., Oterkus, E. (2014). Peridynamic Theory and Its Applications, Springer Science+Business Media New, New York, Dol:10.1007/978-1-4614-8465-3.

[50] Madenci, E., Oterkus, S. (2016). Ordinary state-based peridynamics for plastic deformation according to vonMises yield criteria with isotropic hardening. Journal of the Mechanics and Physics of Solids, vol. 86, p. 192-219, D0l:10.1016/j. jmps.2015.09.016.

[51] Van Le, Q., Bobaru, F. (2018). Objectivity of state-based peridynamic models. Journal of Elasticity, vol. 131, no. 1, p. 1-17, D0l:10.1007/s10659-017-9641-6.

[52] Chowdhury, S. R., Debasish, P. R., Reddy, J. (2019). A modified peridynamics correspondence principle: Removal of zero-energy deformation and other implications. Computer
Methods in Applied Mechanics and Engineering, vol. 346, p. 530-549, D0l:10.1016/j.cma.2018.11.025.

[53] Breitzman, T., Dayal, K. (2018). Bond-level deformation gradients and energy averaging in peridynamics. Journal of the Mechanics and Physics of Solids, vol. 110, p. 192-204, D0I:10.1016/j.jmps.2017.09.015.

[54] Chen, H. (2018). Bond-associated deformation gradients for peridynamic correspondence model. Mechanics Research Communications, vol. 90, p. 34-41, D0l:10.1016/j. mechrescom.2018.04.004.

[55] Silling, S. A., Bobaru, F. (2005). Peridynamic modeling of membranes and fibers. International Journal of NonLinear Mechanics, vol. 40, p. 395-409, D0l:10.1016/j. ijnonlinmec.2004.08.004.

[56] Kilic, B., Agwai, A., Madenci, E. (2009). Peridynamic theory for progressive damage prediction in center-cracked composite laminates. Composite Structures, vol. 90, p. 141-151, DOI:10.1016/j.compstruct.2009.02.015.

[57] Diyaroglu, C., Oterkus, E., Madenci, E., Rabczuk, T., Siddiq, A. (2016). Peridynamic modeling of composite laminates under explosive loading. Composite Structures, vol. 144, p. 14-23, DOI:10.1016/j.compstruct.2016.02.018.

[58] Hu, Y. L., Madenci, E. (2018). Peridynamics for fatigue life and residual strength prediction of composite laminates. Composite Structures, vol. 160, p. 169-184, D0l:10.1016/j. compstruct.2016.10.010.

[59] Ren, B., Wu, C. T., Seleson, P., Zeng, D., Lyu, D. (2018). A peridynamic failure analysis of fiber-reinforced composite laminates using finite element discontinuous Galerkin approximations. International Journal of Fracture, vol. 214, p. 49-68, D0l:10.1007/s10704-018-0317-4.

[60] O'Grady, J., Foster, J. T. (2014). Peridynamic beams: A nonordinary, state-based model. International Journal of Solids and Structures, vol. 51, no. 18, p. 3177-3183, D0I:10.1016/j. ijsolstr.2014.05.014.

[61] Yang, Z., Oterkus, E., Oterkus, S. (2021). Analysis of functionally graded Timoshenko beams by using peridynamics. Journal of Peridynamics and Non-local Modeling, vol. 3, D0l:10.1007/ s42102-020-00044-9.

[62] Ladányi, G., Jenei, I. (2008). Analysis of plastic peridynamic material with RBF meshless method. Periodica Polytechnika, vol. 3, p. 65-77, Dol:10.1556/Pollack.3.2008.3.6.

[63] Lubliner, J. (2008). Plasiticity Theory, Dover Publications, New York.

[64] Mitchell, J. A. (2011). A Non-local, Ordinary, State-Base Plasticity Model for Peridynamics. Albuquerque, New Mexico and Livermore, Dol:10.2172/1018475.

[65] Littlewood, D., Foster, J. T., Boyce, B. (2012). Peridynamic Modeling of Localization in Ductile Metals. Albuquerque. New Mexico and Livermore, D0I:AC04-94AL85000.

[66] Simo, J. C., Hughes, T. J. R. (1998). Computational Inelasticity, Springer, New York.

[67] Kružík, M., Mora-Corral, C., Stefanelli, U. (2018). Quasistatic elastoplasticity via Peridynamics: existence and localization. Continuum Mechanics and Thermodynamics, vol. 30, p. 1155-1184, Dol:10.1007/s00161-018-0671-5. 
[68] Foster, J. T., Silling, S. A., Chen, W. W. (2009). Viscoplasticity using peridynamics. International Journal for Numerical Methods in Engineering, p. 115-132, D0l:10.1002/nme.2725.

[69] Pack, K., Luo, M., Wierzbicki., T. (2014). Sandia fracture challenge: Blind prediction and full calibration to enhance fracture predictability. International Journal of Fracture, vol. 186, D0l:10.1007/s10704-013-9923-3.

[70] Boyce, B. L. (2016). The second Sandia Fracture Challenge: predictions of ductile failure under quasi-static and moderaterate dynamic loading. International Journal of Fracture, vol. 198, no. 1-2, D0l:10.1007/s10704-016-0089-7.

[71] Kramer, S. L. B. (2019). The third Sandia Fracture Challenge: predictions of ductile fracture in additively manufactured metal. International Journal of Fracture, vol. 218, no. 1, p. 1573-2673, DOI:10.1007/s10704-019-00361-1.

[72] Hu, W., Ha, Y. D., Bobaru, F., Silling, S. A. (2012). The formulation and computation of the non-local J-integral in bond-based peridynamics. International Journal of Fracture, vol. 176, no. 2, p. 195-206, D0l:10.1007/s10704-012-9745-8.

[73] Stenström, C., Eriksson, K. (2019). The J-contour integral in peridynamics via displacements. International Journal of Fracture, vol. 216, no. 2, p. 173-183, D0l:10.1007/s10704019-00351-3.

[74] Panchadhara, R., Gordon, P. A. (2016). Application of peridynamic stress intensity factors to dynamic fracture initiation and propagation. International Journal of Fracture, vol. 201, no. 1, p. 81-96, D0l:10.1007/s10704-016-0124-8.

[75] Taylor, M., Steigmann, D. J. (2013). A two-dimensional peridynamic model for thin plates. Mathematics and Mechanics of Solids, vol. 20, no. 8, p. 998-1010, DOl:10.1177/1081286513512925.

[76] Chowdhury, S. R., Roy, P., Roy, D., Reddy, J. N. (2016). A peridynamic theory for linear elastic shells. International Journal of Solids and Structures, vol. 84, D0l:110-132, 10.1016/j.ijsolstr.2016.01.019.

[77] Qi, Z., Shaofan, L., Zao., A-M., Yuxiang, P., Jiale, Y. (2021). A peridynamic Reissner-Mindlin shell theory. Numerical Methods in Engineering, vol. 122, p. 122-147, D0l:10.1002/nme.6527.

[78] Kilic, B., Madenci, E. (2009). Prediction of crack paths in a quenched glass plate by using peridynamic theory. International Journal of Fracture, vol. 156, p. 165-177, D0I:10.1007/s10704-009-9355-2.

[79] Hu, Y. L., Madenci, E., Phan, N. (2018). Peridynamic Modeling of Cracking in Ceramic Matrix Composites. Proceedings of the 17th International Conference on New Trends in Fatigue and Fracture, p. 345-354, Dol:10.1007/978-3-319-70365-7_40.

[80] Bobaru, F., Ha, Y., Hu, W. (2012). Damage progression from impact in layered glass. Central European Journal of Engineering, vol. 2, p. 551-561, D0I:10.2478/s13531-0120020-6.

[81] Hou, D., Zhang, W., Wang, P., Ma, H. (2019). Microscale peridynamic simulation of damage process of hydrated cement paste subjected to tension. Construction and Building Materials, vol. 228, p. 117053, D0l:10.1016/j. conbuildmat.2019.117053.

[82] Cheng, Z., Zhang, G., Wang, Y., Bobaru, F. (2015). A peridynamic model for dynamic fracture in functionally graded materials. Composite Structures, vol. 133, p. 529-546, DOI:10.1016/J.compstruct.2015.07.047.

[83] Bobaru, F., Duangpanya, M. (2012). A peridynamic formulation for transient heat conduction in bodies with evolving discontinuities. Journal of Computational Physics, vol. 231, p. 2764-2785, D0l:10.1016/j.jcp.2011.12.017.

[84] Oterkus, S., Madenci, E., Agwai, A. (2014). Fully coupled peridynamic thermomechanics. Journal of the Mechanics and Physics of Solids, vol. 64, p. 1-23, D0l:10.1016/j. jmps.2013.10.011.

[85] Madenci, E., Oterkus, S. (2017). Ordinary state-based peridynamics for thermoviscoelastic deformation. Engineering Fracture Mechanics, vol. 175, p. 31-45, D0l:10.1016/j. engfracmech.2017.02.011.

[86] Amani, J. Oterkus, E., Areias, P., Zi, P., Nguyen-Thoi, T., Rabczuk, T. (2016). A non-ordinary state-based peridynamics formulation for thermoplastic fracture. International Journal of Impact Engineering, vol. 87, p. 83-94, D0l:10.1016/j. ijimpeng.2015.06.019.

[87] Bazazzadeh, S., Mossaiby, F., Shojaei, A. (2020). An adaptive thermo-mechanical peridynamic model for fracture analysis in ceramics. Engineering Fracture Mechanics, vol. 223, p. 106708, D0l:10.1016/j.engfracmech.2019.106708.

[88] Liu, W., Hong, J.-W. (2012). A coupling approach of discretized peridynamics with finite element method. Computer Methods in Applied Mechanics and Engineering, vol. 245-246, p. 163175, D0I:10.1016/j.cma.2012.07.006.

[89] Wildman, R. A., O'Grady, J. T., Gazonas, G. A. (2017). A hybrid multiscale finite element/peridynamics method. International Journal of Fracture, vol. 207, p. 41-53, D0l:10.1007/s10704017-0218-y.

[90] Giannakeas, I. N., Papathanasiou, T. K., Fallah, A. S., Bahai, H. (2020). Coupling XFEM and Peridynamics for brittle fracture simulation. Computational Mechanics, vol. 66, no. 3, p. 683705, D0l:10.1007/s00466-020-01872-8.

[91] Fan, H., Li, S. (2017). Parallel peridynamics-SPH simulation of explosion induced soil fragmentation by using OpenMP. Computational Particle Mechanics, vol. 4, p. 199-211, DOI:10.1007/s40571-016-0116-5.

[92] Brothers, M. D., Foster, J. T., Millwater, H. R. (2014). A comparison of different methods for calculating tangentstiffness matrices in a massively parallel computational peridynamics code. Computer Methods in Applied Mechanics and Engineering, vol. 279, p. 247-267, Dol:10.1016/j. cma.2014.06.034.

[93] Diehl, P., Schweitzer, M. A. (2015). Efficient neighbor search for particle methods on GPUs. Barth, T. J., Griebel, M., Keyes, D. E., Nieminen, R. M., Roose, D., Schlick, T. (Eds.) Meshfree Methods for Partial Differential Equations VII. Lecture Notes in Computational Science and Engineering, Springer, Cham, DOI:10.1007/978-3-319-06898-5_5.

[94] Meo, D. D., Oterkus, E. (2017). Finite element implementation of a peridynamic pitting corrosion damage model. Ocean Engineering, vol. 135, p. 76-83, D0l:10.1016/j. oceaneng.2017.03.002. 